\title{
Impact of the UK COVID-19 pandemic on HbA1c testing and its implications for diabetes diagnosis and management
}

Letter to the Editor

Diabetes mellitus (DM) is a risk factor for poor outcome in patients with COVID-19. ${ }^{1}$ However, the focus on mitigating the effects of SARS-CoV-2 has resulted in many routine healthcare services, including blood test monitoring in conditions such as DM, being disrupted.

We recently explored the impact of the COVID-19 pandemic on DM diagnosis and management using routinely collected laboratory data on the key DM test, glycated haemoglobin (HbA1c).

We extracted HbA1c data from clinical laboratory information systems at the University Hospitals of North Midlands (UHNM), St. Helens \& Knowsley Hospitals (STHK), Salford Royal Foundation Trust (SRFT), Cambridge University Hospitals (CUH) and Warrington \& Halton Hospitals (WHH) from October 2017-September 2020 (representing 3.3 million people; 4.8\% of the UK population). We were particularly interested to compare the periods before and after the United Kingdom (UK) lockdown on 23 March 2020 and the related curtailment of usual National Health Service (NHS) activities in relation to routine programmed care, in order to focus resources on management of those people acutely unwell with COVID-19.

From these data ( 3 million tests), we calculated the monthly number of missed diagnostic/monitoring tests between 23 March-30 September 2020). We compared the period leading up to 23 March 2020 (UK Lockdown) to the period post 23 March 2020.

We showed that HbA1c tests dropped by $82 \%-88 \%$ in April 2020 and had not reached expected volumes by September (Figure 1). During the 6-month period, in people with DM or at risk of DM, 206422 monitoring tests were missed. Of these, 23466 (11.4\%) had previous $\mathrm{HbA1c}$ values $\geq 59 \mathrm{mmol} / \mathrm{mol}$. The testing delay in this group would, on average, result in a mean increase in $\mathrm{HbA} 1 \mathrm{c}$ of $5.7 \mathrm{mmol} / \mathrm{mol}$ (95\% confidence interval $(\mathrm{Cl}) 5.2-6.2 \mathrm{mmol} / \mathrm{mol}$ ) over and above that expected if monitoring were performed according to NICE guidance. ${ }^{2-4}$ This estimate is based on the analysis of $400497 \mathrm{HbA} 1 \mathrm{c}$ tests in 79409 individuals as previously described. ${ }^{4}$ We found in that analysis that testing outside guidance on $\mathrm{HbA} 1 \mathrm{c}$ monitoring frequency, is associated with a significant detrimental effect on diabetes control.

There were also an estimated 81245 missed diagnostic tests. Of these, $\sim 6105$ (7.5\%) would be expected to be in the prediabetes range $(42-47 \mathrm{mmol} / \mathrm{mol})$ and $\sim 3633(4.5 \%)$ with the diabetes range ( $\geq 48 \mathrm{mmol} / \mathrm{mol}$ ), with 1333 of these having $\mathrm{HbA} 1 \mathrm{c}$ values of $\geq 76 \mathrm{mmol} / \mathrm{mol}$.

Extrapolating this to the UK population, these data equate to missed monitoring tests in 489000 people with sub-optimallycontrolled diabetes, leading to missed glycaemic control targets with associated increased risk of complications, including symptomatic cardiovascular disease and renal impairment, with their associated excess mortality risk. ${ }^{5}$ These data also equate to 127000 missed pre-diabetes and 76000 missed diabetes diagnoses, with consequent delay in lifestyle advice and treatment initialisation as advised by NICE. ${ }^{2,4}$

We have previously shown that $\mathrm{HbA1c}$ testing at a 3 monthly interval was associated with a $3.8 \%$ reduction in $\mathrm{HbA} 1 \mathrm{c}$ compared with a $1.5 \%$ increase observed with annual testing. Compared with annual monitoring, 3-monthly testing was associated with a halving of the proportion showing a significant rise in $\mathrm{HbA1c}$ (7-10 vs $15 \%$ $20 \%){ }^{4}$ Thus, any perturbation of the system that results in disruption of testing protocols will likely result in many patients drifting above target glycaemia and in many of those above target $\mathrm{HbA1c}$, remaining at that level. We accept that treatment decisions by clinicians and patients are based on the combination of blood glucose readings (whether by standard finger prick or by continuous blood glucose monitoring) plus HbA1c.

This is not a worse case scenario, but rather based on real world large data in which all the factors described above are in play. Our findings, in keeping with those of a recent study using general practice data, ${ }^{6}$ illustrate the widespread collateral impact of implementing measures to mitigate the impact of COVID-19 in people with, or being investigated for DM. Ironically, failure to focus of the wider implications for people with DM and other groups with long-term conditions, may place them at increased risk of poor outcomes from SARS-CoV-2 infection itself, irrespective of the implications for their longer term health prospects.

This is an open access article under the terms of the Creative Commons Attribution-NonCommercial License, which permits use, distribution and reproduction in any medium, provided the original work is properly cited and is not used for commercial purposes.

(c) 2021 The Authors. International Journal of Clinical Practice published by John Wiley \& Sons Ltd 


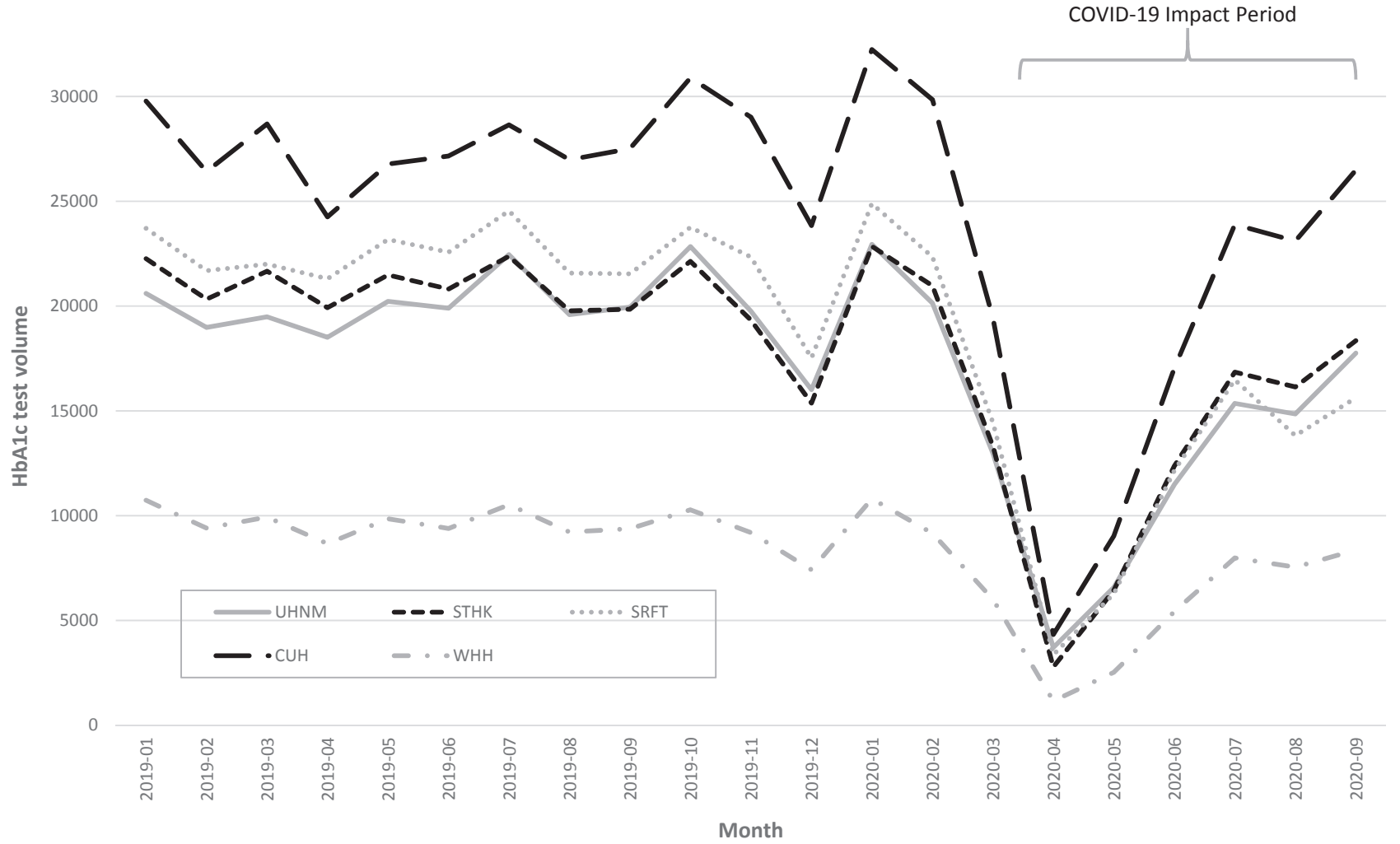

FIGURE 1 Month-by-month HbA1c test numbers across the five sites prior to and during the COVID-19 Impact Period

\author{
David Holland ${ }^{1}$ \\ Adrian H. Heald ${ }^{2,3}$ iD \\ Mike Stedman ${ }^{4}$ \\ Lewis Green ${ }^{5}$ \\ Jonathan Scargill ${ }^{6}$ \\ Christopher J. Duff ${ }^{7,8}$ \\ Fahmy W.F. Hanna 9,10 \\ Pensee $\mathrm{Wu}^{7,11}$ \\ Ian Halsall ${ }^{12}$ \\ Neil Gaskell ${ }^{13}$ \\ Anthony A. Fryer 7,8 (iD)
}

${ }^{1}$ The Benchmarking Partnership, Alsager, UK

${ }^{2}$ Department of Diabetes and Endocrinology, Salford Royal NHS Foundation Trust, Salford, UK

${ }^{3}$ The School of Medicine and Manchester Academic Health Sciences Centre, The University of Manchester, Manchester, UK

${ }^{4}$ Res Consortium, Andover, UK

${ }^{5}$ St. Helens \& Knowsley Teaching Hospitals NHS Trust, Whiston

Hospital, Prescot, UK

${ }^{6}$ Department of Clinical Biochemistry, The Royal Oldham Hospital, The Northern Care Alliance NHS Group, Oldham, UK ${ }^{7}$ School of Medicine, Keele University, Keele, Staffordshire, UK ${ }^{8}$ Department of Clinical Biochemistry, University Hospitals of North Midlands NHS Trust, Stoke-on-Trent, Staffordshire, UK

${ }^{9}$ Department of Diabetes and Endocrinology, University
Hospitals of North Midlands NHS Trust, Stoke-on-Trent, Staffordshire, UK

${ }^{10}$ Centre for Health \& Development, Staffordshire University, Stoke-on-Trent, Staffordshire, UK

${ }^{11}$ Department of Obstetrics \& Gynaecology, University Hospitals of North Midlands NHS Trust, Stoke-on-Trent, Staffordshire, UK

${ }^{12}$ Department of Clinical Biochemistry, Cambridge University Hospitals NHS Foundation Trust, Addenbrooke's Hospital, Cambridge, UK

${ }^{13}$ Department of Pathology, Warrington \& Halton Teaching Hospitals NHS Foundation Trust, Warrington, UK

\section{Correspondence}

Adrian Heald, Department of Diabetes and Endocrinology, Salford Royal NHS Foundation Trust, Salford, The School of Medicine and Manchester Academic Health Sciences Centre, The University of Manchester, Manchester, UK. Email: adrian.heald@manchester.ac.uk

\section{ORCID}

Adrian H. Heald (ID) https://orcid.org/0000-0002-9537-4050

Anthony A. Fryer (iD https://orcid.org/0000-0001-8678-0404

\section{REFERENCES}

1. Muniangi-Muhitu H, Akalestou E, Salem V, Misra S, Oliver NS, Rutter GA. Covid-19 and Diabetes: A Complex Bidirectional Relationship. Front Endocrinol (Lausanne). 2020;11:582936. 
2. National Institute for Health and Care Excellence (NICE). Type 2 diabetes in adults: management NICE guideline [NG 28]. 2015. https://www.nice.org.uk/guidance/ng28. Accessed November 13, 2020.

3. Driskell OJ, Holland D, Waldron JL, et al. Reduced testing frequency for glycated hemoglobin, $\mathrm{HbA1c}$, is associated with deteriorating diabetes control. Diabetes Care. 2014;37:2731-2737.

4. Public Health Guideline [PH38]. National Institute for Health and Care Excellence (NICE). Type 2 diabetes: prevention in people at high risk; 2012. https://www.nice.org.uk/guidance/ph38. Accessed November 13, 2020.

5. Klein KR, Buse JB. The trials and tribulations of determining HbA1c targets for diabetes mellitus. Nat Rev Endocrinol. 2020;16:717-730.

6. Carr MJ, Wright AK, Leelarathna L, et al. Impact of COVID-19 on the diagnoses, $\mathrm{HbA1c}$ monitoring and mortality in people with type 2 diabetes: a UK-wide cohort study involving 13 million people in primary care. https://www.medrxiv.org/content/10.1101/2020.10.25.20200 675v1.full.pdf. Accessed November 13, 2020. 